Andrzej Bonasewicz

\title{
LES POLONAIS EN LITUANIE ET EN LETTONIE
}

Les Polonais qui habitent l'actuelle Lituanie et Lettonie sont autochtones, présents sur ces territoires depuis quelques centaines d'années.

Les Slaves, et parmi eux certaines tribus venant de Pologne, apparurent déjà au $\mathrm{VI}^{\mathrm{e}}$ siècle sur les terres du futur Grand Duché de Lituanie. Plus tard, nombre de Polonais capturés lors des raids contre la Pologne, y ont été installés. Après l'union personnelle avec la Pologne en 1385 plusieurs ecclésiastiques, administrateurs et hommes de cours polonais sont arrivés. Cette émigration, surtout de nobles, s'est accrue après l'union de Lublin en 1569 qui a réuni les deux pays en un seul. Les Polonais s'établirent également en Livonie (Sud de l'actuelle Lettonie), incorporée en partie à la République des deux nations en 1570. Dans le Grand Duché les Biélorussiens dominaient et le russe servait de langue officielle. Wilno (Vilnius), après la fondation de l'Université par le roi Stefan Batory en 1578, devint un centre d'où rayonnait l'élément polonais. En 1695 le russe fut remplacé par le polonais comme langue officielle; le lituanien était utilisé par une minorité habitant Żmudź (Samogitie), au Nord-Ouest de l'actuelle Lituanie.

Fin XVIII ${ }^{\mathrm{e}}$ siècle, après le partage de la Pologne entre la Russie, la Prusse et l'Autriche, la Lituanie et la Lettonie sont devenues russes. Commence alors une politique antipolonaise, dirigée contre la noblesse et les habitants des villes, et favorisant les paysans, généralement Lituaniens et Lettons. Après l'échec de l'insurrection de 1863, l'abolition du servage et l'instauration de la propriété paysanne, des mouvements nationalistes lituanien et letton ont commencé à se manifester. En conséquence, une nouvelle intelligentsia apparaît, qui contribue à la formation de la Lituanie et de la Lettonie indépendantes en 1918.

Les premiers recensements qui comportent des statistiques par nationalité datent de 1897 et 1909. Le gouvernorat de Kaunas (Kowno) et en partie celui de Vilnius (Wilno) correspondent à l'actuelle Lituanie, tandis que Kurzeme (Curlande) et Vidzeme (Liflande) - à l'actuelle Lettonie. Le tableau 1 indique le nombre de Polonais dans ces unités administratives.

Dans les villes, le pourcentage de Polonais était plus élevé que dans les 


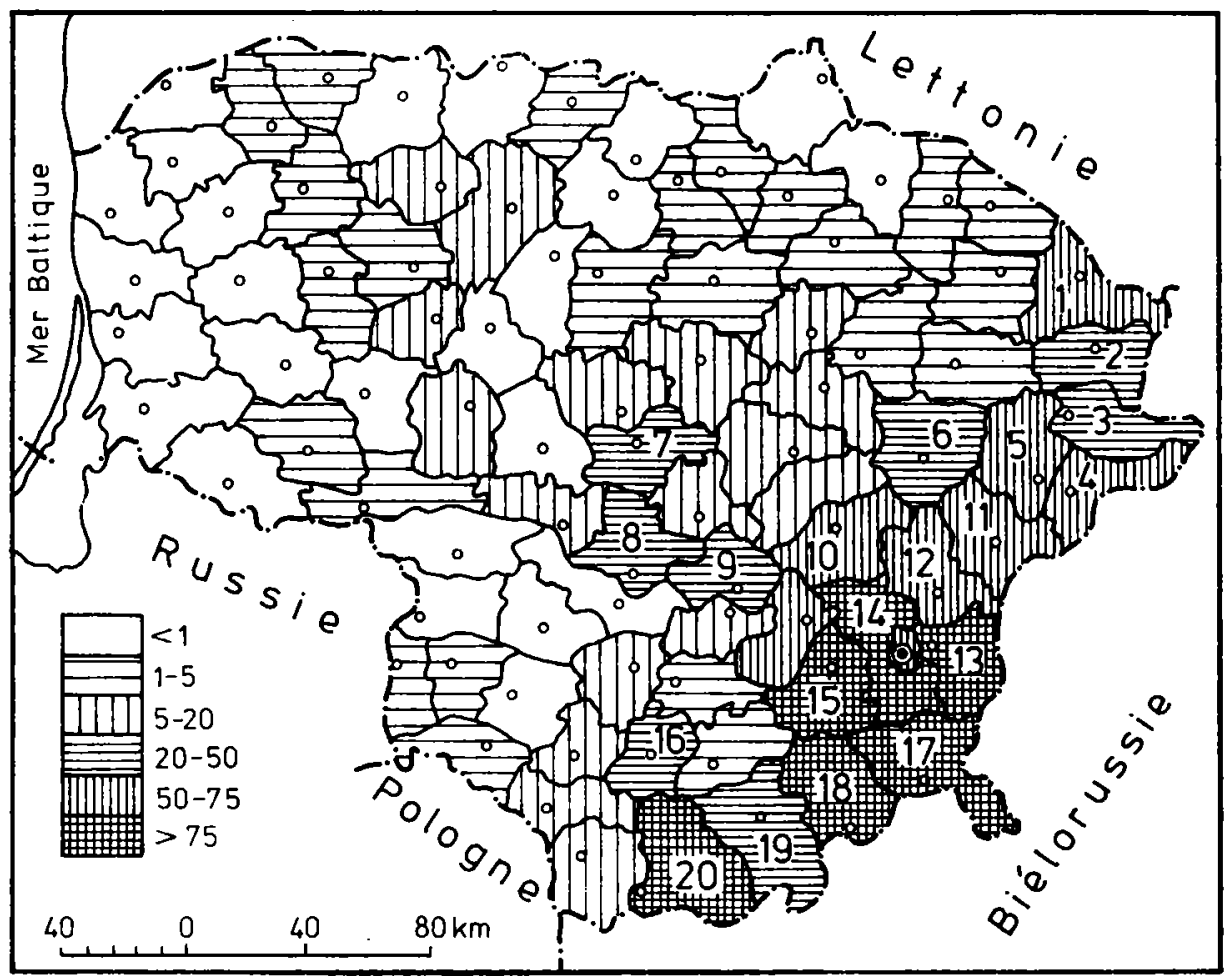

Fig. 1. Le pourcentage de Polonais dans les circonscriptions de la Lituanie, en 1923.

1 -Zarasai, 2 - Dukštos, 3 - Ignalina, 4 - Svenčionys, 5 - Svenčionele, 6 - Moletai, 7 Kiedainiai, 8 - Kaunas, 9 - Kaišadorys, 10 - Sirvintai, 11 - Pabrade, 12 - Nemenčine, 13 - Nauijoi Vilnia, 14 - Vilnius, 15 - Trakai, 16 - Alytus, 17 - Salčininkai, 18 Eišiskis, 12 - Varena, 20 - Druskininkai

campagnes. Les Lituaniens dominaient dans le gouvernorat de Kaunas (66\%), les Biélorussiens - dans celui de Vilnius (56\%). La diminution de la présence polonaise dans le gouvernorat de Kaunas résultait surtout des répressions de la part des autorités russes et de la part des nationalistes lituaniens. Nombre de Polonais émigraient dans le gouvernorat de Vilnius, où les Biélorussiens n'avaient pas d'aspirations nationalistes. En 1916, d'après le recensement effectué par les Allemands, les Polonais constituaient $6,9 \%$ de la population de la région administrative de Kaunas (35\% dans la ville de Kaunas, $73 \%$ dans la circonscription de Zarasai, $40-50 \%$ dans celles de Moletai et Kaišiadorys) et $34,9 \%$ dans la région de Vilnius ( $50 \%$ dans la ville de Vilnius, $91 \%$ dans la circonscription de Vilnius, $61 \%$ dans celle de Švenčionys, $56 \%$ à Trakai). A Vilnius, les Juifs étaient les plus nombreux $(43,5 \%)$, les Lituaniens ne représentaient que $2,6 \%$ de la population.

En 1923, en Lituanie indépendante, le recensement de la population révélait 64105 Polonais (seulement $3,2 \%$ du total), mais ces chiffres sont bien sûr sujets à caution car les listes électorales polonaises ont rassemblé 


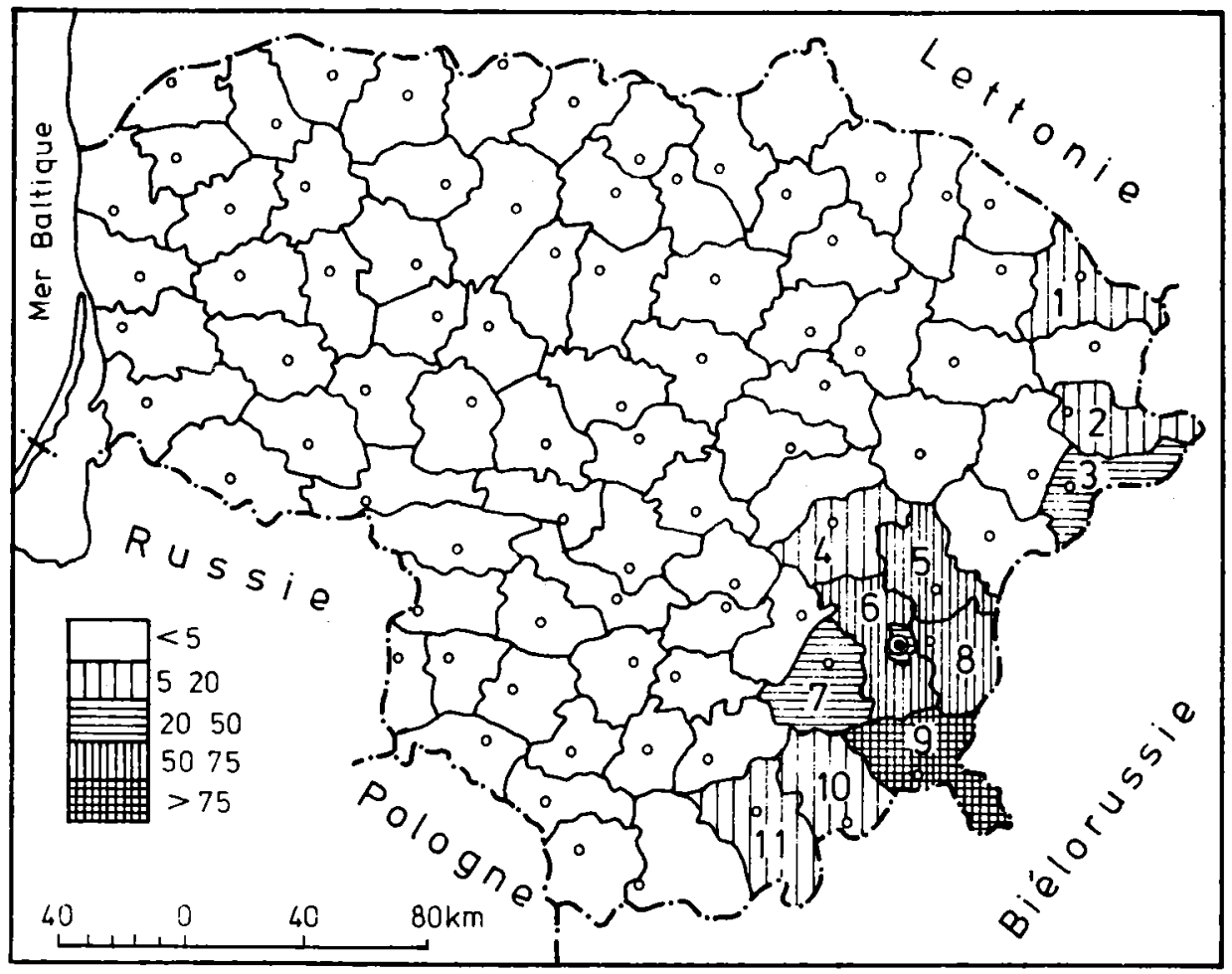

Fig. 2. Le pourcentage de Polonais dans les circonscriptions de la Lituanie, en' 1989.

1 -Zarasai, 2 - Ignalina, 3 - Švenčionys, 4 - Širvintai, 5 - Nemenčine, 6 - Vilnius, 7 Trakai, 8 - Naujoi Vilnia, 9-Salčininkai, 10 - Eišiskis, 11 - Varena

en même temps 202026 voix. La carte indique la participation des Polonais dans les circonscriptions de la Lituanie et dans la région de Wilno, estimée selon l'Institut d'Études Internationales de Varsovie en 1929. Cette participation était la plus élevée dans la circonscription de Kaunas (31\%), Kaišadorys (29\%), Ukmerge (22\%), Kiedainiai (21\%) et Zarasai (16\%); dans les villes de la région de Wilno elle atteignait $60 \%$, à la campagne $-63 \%$.

D'après le recensement de 1937 , le nombre de Polonais en Lettonie s'élevait à 64884 personnes (3,1\% du total de la population); parmi eux, 49,7\% habitaient l'ancienne Latgalie polonaise - surtout à Daugavpils, Ilukste, Ludza, Rezekne. Avec les travailleurs saisonniers, ce nombre était estimé à 90-95 000 personnnes.

Après le déclenchement de la Deuxième guerre mondiale, le 10 octobre 1939 la partie occidentale de la région de Wilno $\left(9500 \mathrm{~km}^{2}\right)$, avec la ville de Wilno, a été incorporée à la Lituanie. Le nombre de Polonais dans ce pays a donc considérablement augmenté, puisque ces territoires étaient habités en majorité par la population polonaise. D'après le recensement effectué par les Allemands en 1942, ce nombre s'élevait à 335000 (12,1\% de la popula- 
tion totale), sans compter les déportés à l'intérieur de l'URSS en 1939 et 1941, et en Allemagne, surtout comme travailleurs forcés. Les Polonais contituaient $71,9 \%$ de la population de Wilno, $43,5 \%$ de sa circonscription

Tableau 1

Les Polonais en Lituanie, en 1897 et 1906

\begin{tabular}{|c|r|r|r|r|}
\hline \multirow{2}{*}{ Unité administrative } & \multicolumn{2}{|c|}{1897} & \multicolumn{2}{c|}{1906} \\
\cline { 2 - 5 } & nombre & $\begin{array}{c}\text { pourcentage } \\
\text { de la popula- } \\
\text { tion totale }\end{array}$ & nombre & $\begin{array}{c}\text { pourcentage } \\
\text { de la popula- } \\
\text { tion totale }\end{array}$ \\
\hline Gouvernorat de Kaunas & 138408 & 9,1 & 57837 & 3,5 \\
circonscr. de Zarasai & 18577 & 8,9 & 5775 & 2,8 \\
$" \quad$ de Kaunas & 51825 & 24,8 & 20916 & 8,4 \\
$" \quad$ de Panevežys & 14460 & 6,6 & 9414 & 4,1 \\
$" \quad$ de Raseiniai & 12974 & 5,6 & 3652 & 1,5 \\
$" \quad$ de Sauliai & 14917 & 6,3 & 5939 & 2,4 \\
$" \quad$ de Teisliai & 2771 & 1,5 & 253 & 0,1 \\
$" \quad$ de Ukmerge & 22884 & 10,1 & 11888 & 4,5 \\
Gouvernorat de Vilnius & 105402 & 11,3 & 248016 & 24,6 \\
(partie Ouest) & & & & \\
circonscr. de Švenčionys & 10321 & 6,0 & 5547 & 2,9 \\
$" \quad$ de Trakai & 22795 & 11,4 & 52781 & 22,0 \\
$" \quad$ de Vilnius & 25290 & 7,2 & 112188 & 47,5 \\
ville de Vilnius & 46996 & 33,5 & 77500 & 37,8 \\
Kurzeme & 19700 & 2,9 & & \\
Vidzeme & 15100 & 1,2 & & \\
\hline
\end{tabular}

Tableau 2

Les Polonais en Lettonie en 1930 et 1937

\begin{tabular}{|l|r|r|}
\hline \multicolumn{1}{|c|}{ Région } & 1930 & \multicolumn{1}{c|}{1937} \\
\hline Riga & 17323 & 18011 \\
Viodeme & 1962 & 2039 \\
Kurzeme & 3727 & 3875 \\
Zemgale & 9513 & 9892 \\
Latgale & 32159 & 33432 \\
\hline
\end{tabular}

Après la Deuxième guerre mondiale, d'après les recensements soviétiques, le nombre de Polonais en Lituanie et Lettonie, annexées à l'URSS, diminuait constamment. Dans le cadre de l'action du "transfert" de la population, 180000 Polonais ont quitté la Lituanie (dont 108000 la ville de Vilnius) en 1945-47, 80000 autres en 1955-57. Pendant les recensements, l'effectif de Polonais était toujours délibérément réduit; d'autre part, nombre de Polonais ne révélaient pas leur nationalité pour améliorer leur situation. En 1959, les Polonais représentaient 8,5\% de la population, en $1970-7,7 \%$, en $1979-7,3 \%$, en $1989-7,0 \%$. D'après ce dernier recense- 
ment général leur nombre s'élevait à 258000 , tandis que d'après le recensement suivant les communes - $355500(9,13 \%)$. Ce nombre est reconnu par l'Union des Polonais en Lituanie - organisation qui compte 15000 membres répartis en 150 associations. Suivant les données de cette Union, en 1990 les Polonais représentaient $18,6 \%$ de la population de la ville de Vilnius (110 000 personnes), 63\% de la population de sa circonscription (60 000), $80 \%$ de celle de Salčininkai (33 000 ). La participation des Polonais était également élevée dans les circonscriptions de Trakai (23,5\%), de Švenčionys $(28,8 \%)$, d'Ignalina $(7,5 \%)$, de Varena (7\%). A Kaunas et à Klaipeda, ils ne représentaient plus que $0,5 \%$ de la population.

La perte d'identité polonaise se fait surtout à cause du manque d'éducation en polonais. Il y avaient 236 écoles polonaises en 1953, 92 seulement en 1987; ce nombre a augmenté jusqu'à 123 en 1991. 11400 enfants polonais ( $25 \%$ du total) y ont été scolarisés. 15 prêtres catholiques polonais exerçaient dans 659 paroisses, parmi lesquelles plus de 50 étaient habitées en majorité par les Polonais. Leur niveau d'instruction est faible: $2,3 \%$ seulement ont terminé les écoles supérieures (d'où la création, en 1991, d'une université polonaise à Vilnius, par les soins de l'Association des chercheurs polonais en Lituanie.

En Lettonie, d'après le recensement de 1989 le nombre de Polonais s'élevait à 61331 (2,3\% de la population). Ils étaient les plus nombreux dans les régions indiquées dans le tableau 3; 98 prêtres catholiques, dont 12 Polonais, y exerf̧aient dans 198 paroisses.

Tableau 3

Les Polonais en Lettonie en 1989; nombre et pourcentage de la population totale

\begin{tabular}{|lrl|}
\hline Région de Riga (sans chef-lieu) & $2564(1,7 \%) ;$ & ville de Riga 16 388 $(1,8 \%)$ \\
Région de Daugavpils " & $5272(11,2 \%) ;$ & ville de Daugavpils $16363(13,1 \%)$ \\
Région de Kraslava & $2489(6,0 \%)$ & \\
Région de Jelgava (sans chef-lieu) & $939(2,4 \%) ;$ & ville de Jelgava 1 258 $(1,7 \%)$ \\
Région de Rezekne & $608(1,4 \%) ;$ & ville de Rezekne 1 146 (2,7\%) \\
\hline
\end{tabular}

Et Lituanie et en Lettonie habitent donc actuellement environ 400000 Polonais autochtones. Certains ne parlent même plus polonais, à cause du manque de contact avec la Pologne, de l'éducation en polonais ou par suite de mariage avec des personnes d'autres nationalités. La politique des autorités visant à diminuer l'importance de l'élément polonais en Lituanie a porté ses fruits surtout dans les territoires qui appartenaient à ce pays avant la Deuxième guerre mondiale; le nombre de Polonais y a considérablement baissé. Ils habitent par contre toujours en densité autour de Vilnius et Salčininkai en Lituanie et à Daugavpils en Lettonie. 


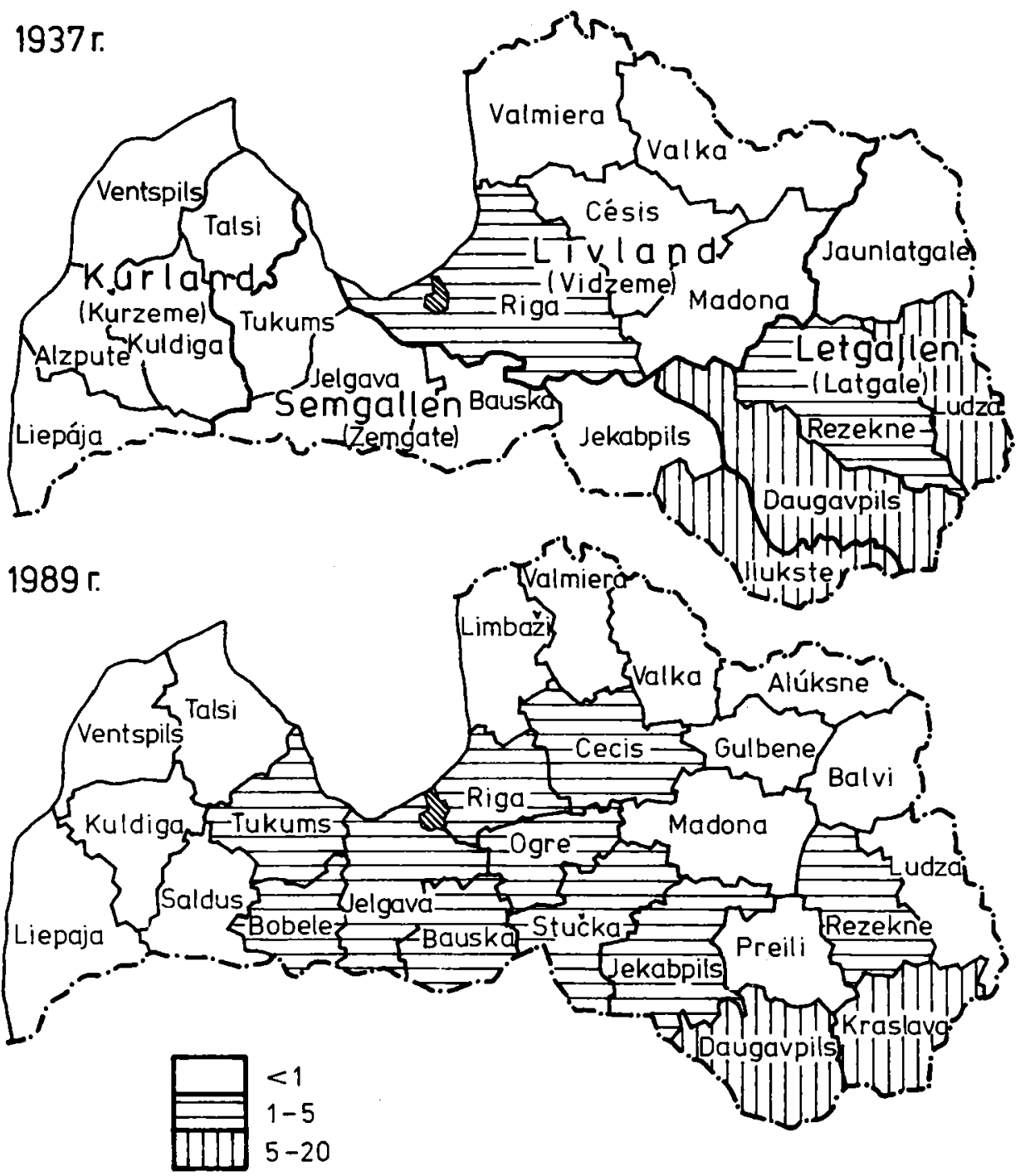

Fig. 3. Le pourcentage de Polonais dans les régions et circonscriptions de la Lettonie, en 1937 et 1989 